\title{
Percutaneous Intervention of a Single Coronary Artery in the Setting of Acute Coronary Syndrome
}

\author{
Mohammady Shahin ${ }^{\mathrm{a}}$, Nooraldaem Yousifa, Thomas F. Luscher ${ }^{\mathrm{a}}$, \\ Slayman Obeid ${ }^{\mathrm{a}, \mathrm{b}}$
}

\begin{abstract}
Performing intervention of a single coronary artery (SCA) is challenging and technically difficult since a severe complication may be catastrophic if occurred. It requires a proper selection of instruments and well experienced operator to perform the intervention. However, a definitive standardization treatment for those patients is difficult; each case should be treated individually, according to the anatomical variations. We present three rare cases of SCA in setting of acute coronary syndrome (ACS) and culprit lesion in right coronary artery (RCA), treated successfully with percutaneous coronary intervention.
\end{abstract}

Keywords: Single coronary artery; Acute coronary syndrome

\section{Introduction}

A single coronary artery (SCA), defined as a coronary artery that arises from the sinus of Valsalva and supplies the entire heart, is congenital and rare, particularly in the absence of structural heart disease [1-5]. The prevalence of single coronary anomalies in the general population is approximately $0.024 \%$ according to Lipton's reports. The various patterns of SCA are difficult to understand. Different classifications present for single coronary artery $[2,5,6]$. Lipton et al systematically classified the different types of SCA [2]. Group I has anatomical course of either a right or left coronary artery. Group II anomalies arise from the proximal part of the normal right or left coronary artery, and cross the base of the heart before assuming the normal position of the inherent coronary artery. Group III describes the anomaly where the left anterior descending (LAD) artery and left circumflex (LCX) artery arise separately from the proximal part of the normal right coronary artery (RCA). Five anatomical subtypes exist, and are classified according to the relationship

Manuscript submitted September 20, 2017, accepted September 27, 2017

aUniversity Heart Center, University Hospital Zurich, Zurich, Switzerland ${ }^{b}$ Corresponding Author: Slayman Obeid, Zurich University Cardiac Center, Zurich, Switzerland. Email: Slayman.Obeid@usz.ch

doi: https://doi.org/10.14740/cr613w of the anomalous coronary artery with the aorta and pulmonary artery, i.e., "anterior," "between," "septal," "posterior," or "combined." In this series, the "septal" subtype was the most common, whereas the "between" subtype was the least common. Another classification adapted from Angelini Circulation 2002 [6] and Wilkins Texas Heart Institute Journal 1988 which classify coronary anomalies in seven groups: 1) origin of both RCA and left main stem (LMS) (separate origins) from the right aortic sinus (a: course of anomalous LMS between aorta and pulmonary artery (PA); b: course of anomalous LMS not between aorta and PA); 2) origin of both coronary arteries (separate origins) from the left aortic sinus (a: course of anomalous RCA between aorta and PA; b: course of anomalous RCA not between aorta and PA); 3) anomalous origin of the circumflex coronary artery from the right aortic sinus (a: course of anomalous LCX between aorta and PA; b: course of anomalous LCX not between aorta and PA); 4) anomalous origin of the LAD artery from the right aortic sinus (a: course of anomalous LAD between aorta and PA; b: course of anomalous LAD not between aorta and PA); 5) single coronary artery (common origin) (a: course of anomalous coronary artery between aorta and PA; b: course of anomalous coronary artery not between aorta and PA); 6) anomalous origin or communication of a coronary artery with a cardiac chamber or major thoracic vessel (a: abnormal origin from the pulmonary artery or one of its major arterial branches; $b$ : abnormal origin from the aorta or one of its major arterial branches; c: abnormal communication of a coronary artery with a cardiac chamber or major thoracic vessel (fistula)); 7) miscellaneous/unclassified.

Most of the cases of SCA are asymptomatic. The malignant variety of SCA in which life-threatening symptom can occur in minor of patients is the type in which the coronary artery courses between the great vessels (aorta and PA). In general, the most convenient examination for suspected coronary anomaly is exercise stress testing; however, this test can be negative or give conflicting results $[5,7]$. On the other hand, cardiac multi-slice computed tomography (MSCT) allows in identifying comprehensive characterizations of coronary artery anomaly and may be useful to gain accurate insight into the culprit artery [8-10]. This test is not necessary when the diagnosis and therapeutic approach are clearly established by coronary angiography. Coronary angiography is commonly used as the standard method to detect coronary artery anomaly if we fail to visualize the origin and total course of a coronary artery anomaly accurately [5], but cardiac MSCT examination 


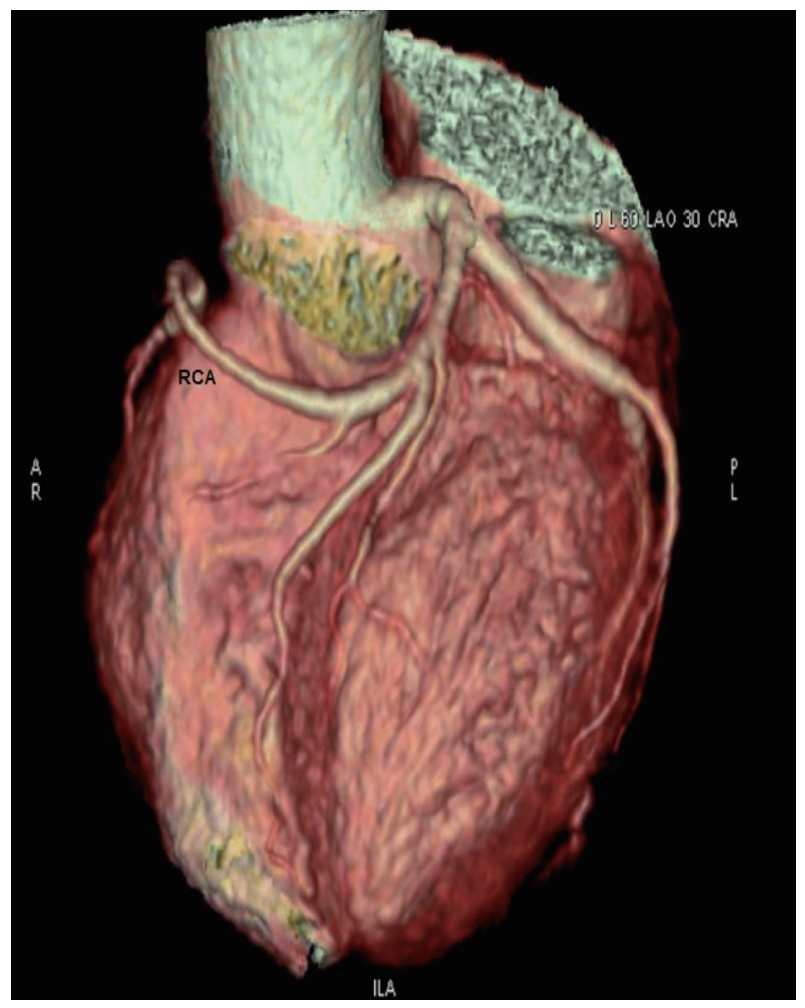

Figure 1. MSCT showing RCA coming from LAD as a continuation of septal branch.

may be useful to better identify the accurate coronary anatomy; moreover, if necessary, it could guide the intervention therapy. Finally, cardiac magnetic resonance imaging provides 3-dimensional image and patient could avoid exposure to ra-

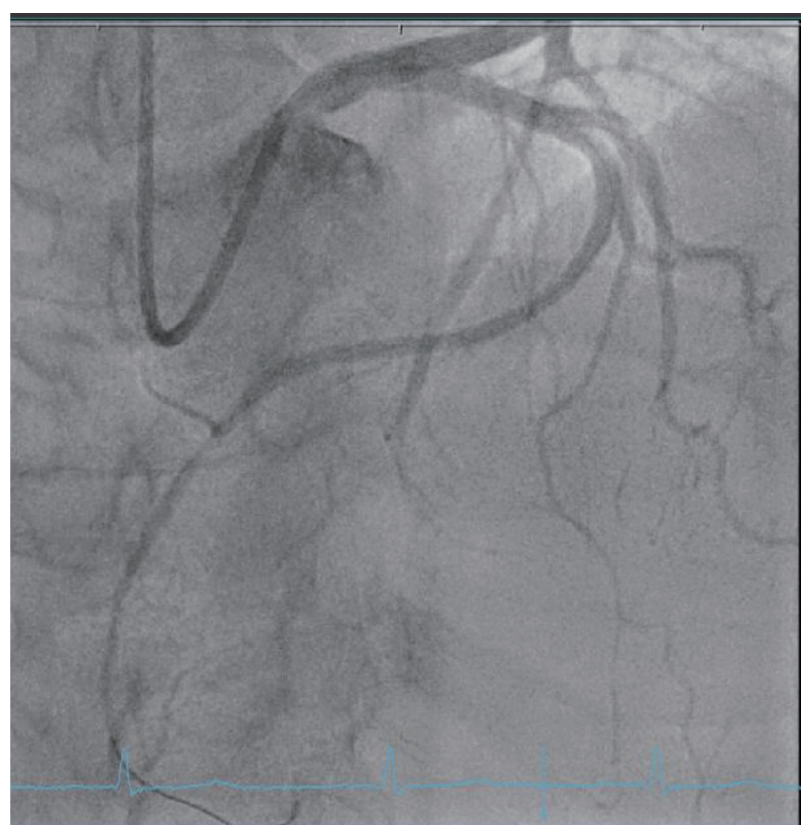

Figure 2. Coronary angiography LAO 8/CR 31 view showing RCA coming from LAD with mid segment RCA stenosis $70-90 \%$.

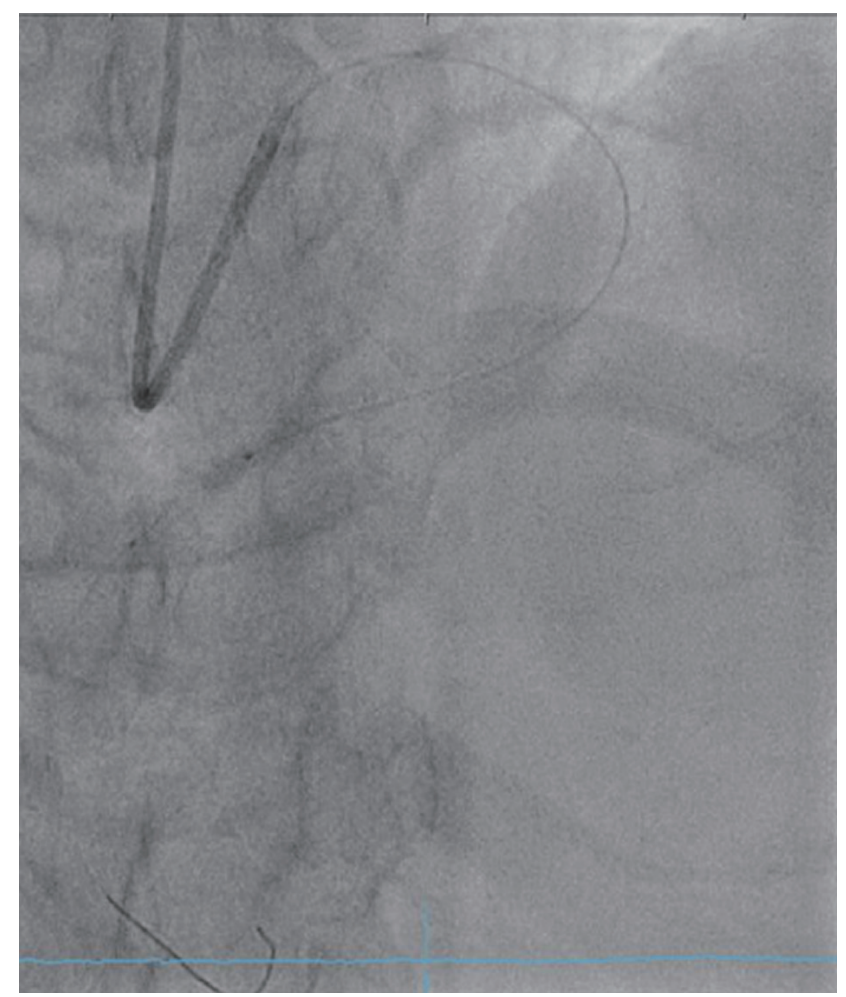

Figure 3. Coronary angiography LAO 8/CR 31 view showing mid RCA stent deployment.

diation.

\section{Case Report}

We present three rare cases of SCA in setting of ACS and culprit lesion in RCA, treated with percutaneous coronary intervention (PCI). The first of them (Figs. 1-4) was a 63-year-old female patient with positive family history of coronary artery disease (CAD), smoker, hypertensive and hyperlipidemic who presented to our department with non-ST-segment elevation myocardial infarction (NSTEMI) and referred to coronary angiography that showed SCA from left coronary sinus with RCA origin from distal LAD (septal branch) with $70-90 \%$ stenosis at mid segment RCA. PCI was done with drug-eluting stent (DES) with good final angiographic results. MSCT was done later on and documented RCA origin from distal LAD segment with open stent at mid segment. The second case (Figs. 5-8) was a 57-year-old male patient with positive family history of CAD, smoker and hyperlipidemic who presented to our department with unstable angina (UA) and referred to coronary angiography that showed SCA from left coronary sinus with RCA origin from left main coronary artery with $70-90 \%$ in-stent restenosis at proximal RCA. MSCT was done and documented RCA origin from left main artery with special course between pulmonary trunk and ascending aorta with 70-90\% in-stent restenosis. Re-PCI was done with DES with good final angiographic results. The third case (Figs. 9-11) was a 59-year-old female patient 


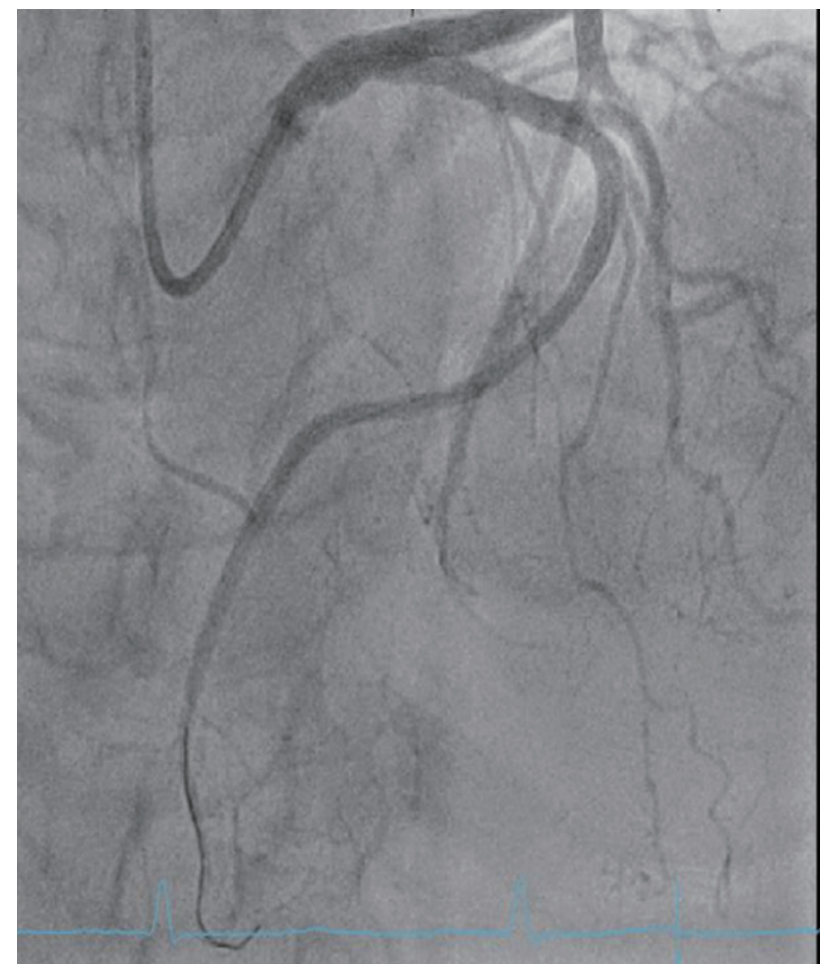

Figure 4. Coronary angiography LAO 8/CR 31 view showing good results after RCA stenting.

with positive family history of CAD, diabetic, hypertensive and hyperlipidemic who presented to our department with NSTEMI and referred to coronary angiography that showed SCA from right coronary sinus with separate LAD and LCX origin from proximal RCA and 99\% stenosis at proximal and

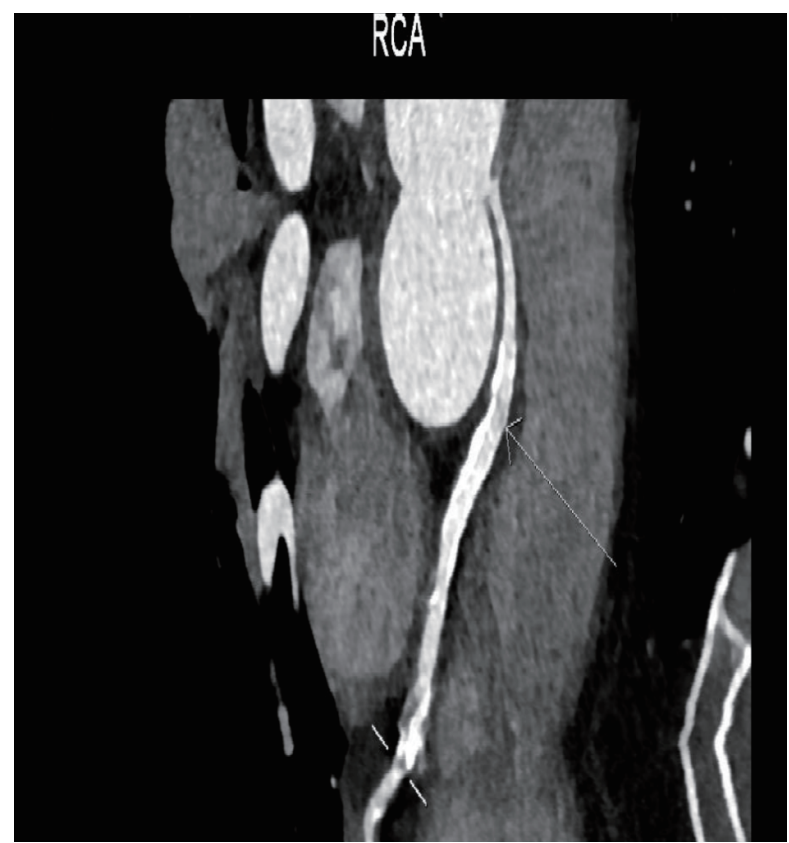

Figure 5. MSCT showing RCA coming from left main coronary artery.

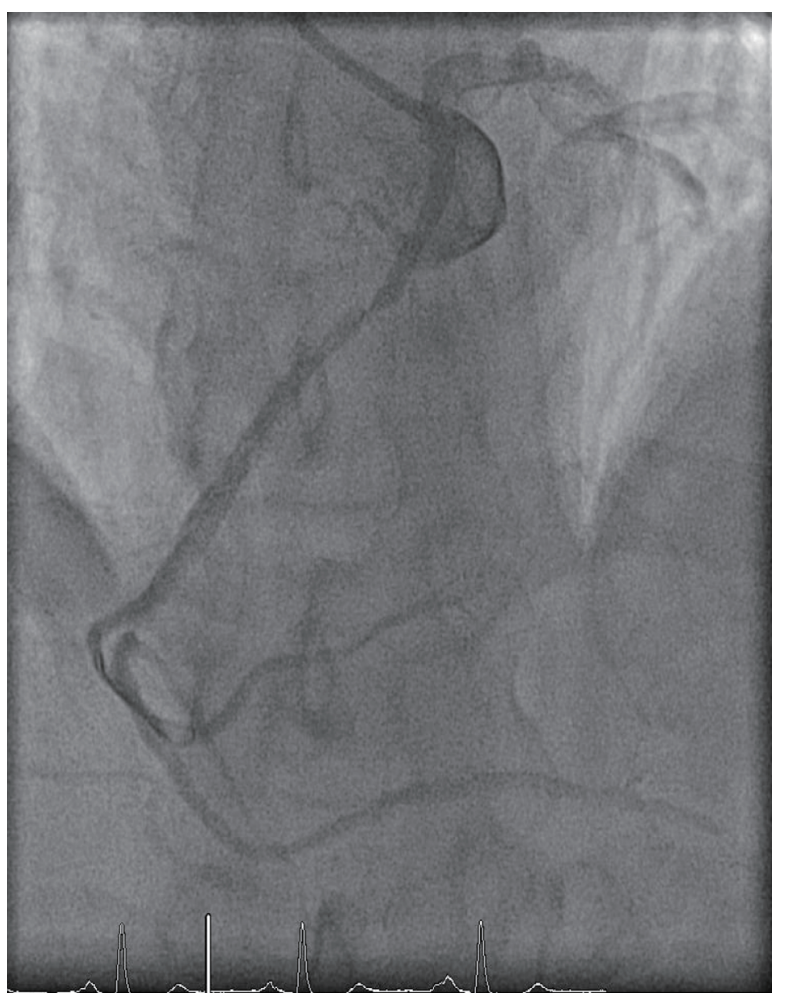

Figure 6. Coronary angiography LAO 6/CR 36 view showing RCA coming from LM with $90 \%$ in-stent restenosis at proximal segment.

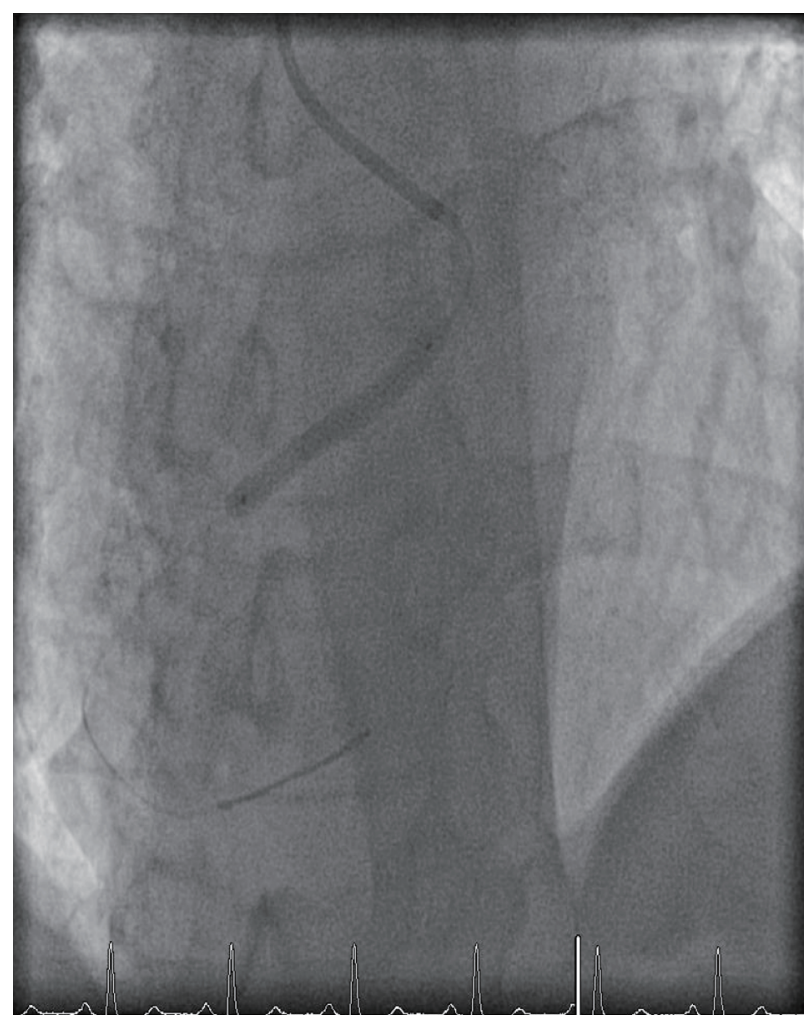

Figure 7. Coronary angiography LAO 6/CR 36 view showing proximal RCA stent deployment. 


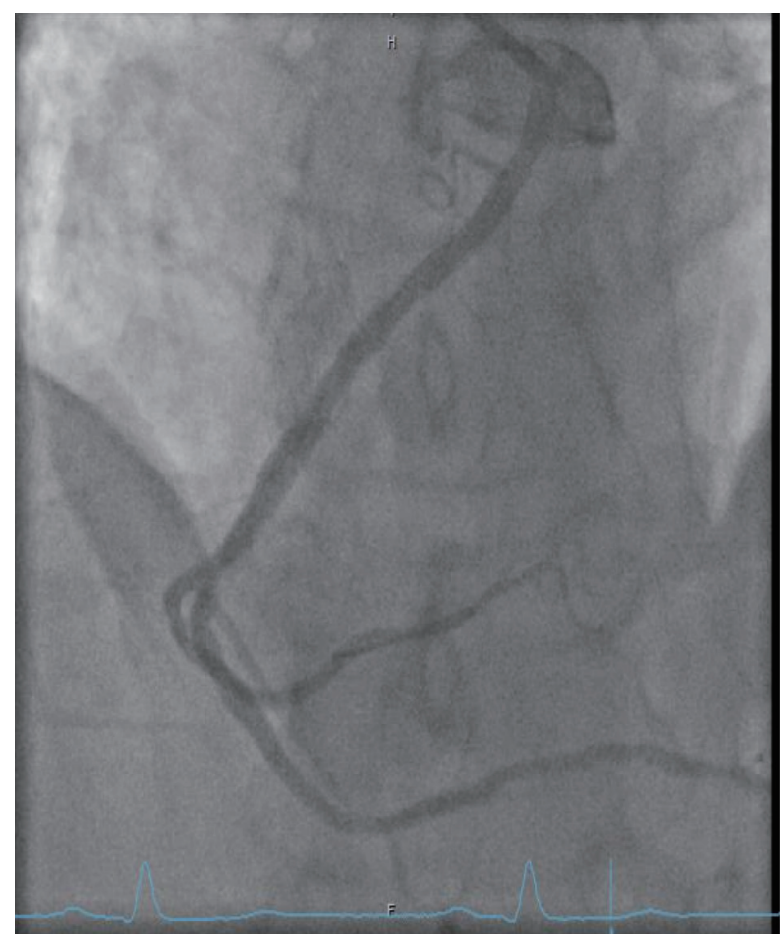

Figure 8. Coronary angiography LAO 6/CR 36 view showing good final results after proximal RCA stent deployment.

mid RCA that was treated with bare metal stent (BMS) and represented after 1 year with in-stent restenosis and DES was used.

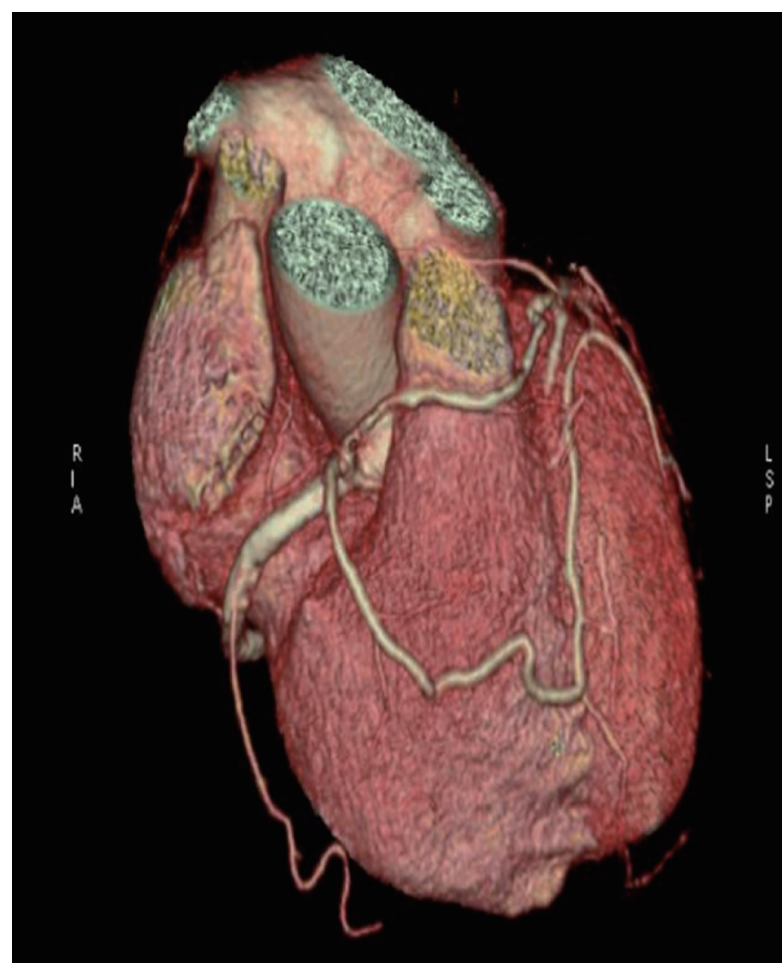

Figure 9. MSCT showing $L A D$ and RCX coming from proximal RCA.

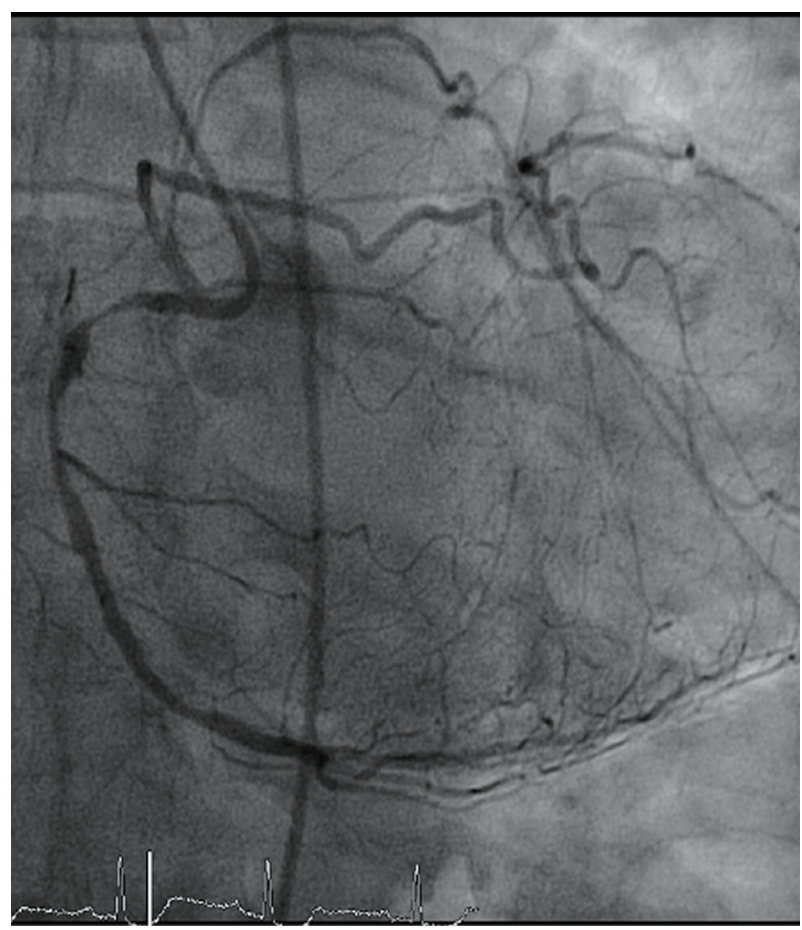

Figure 10. Coronary angiography RAO 20/CA 28 view showing LAD and LCX coming from proximal RCA with $99 \%$ stenosis at proximal and mid RCA.

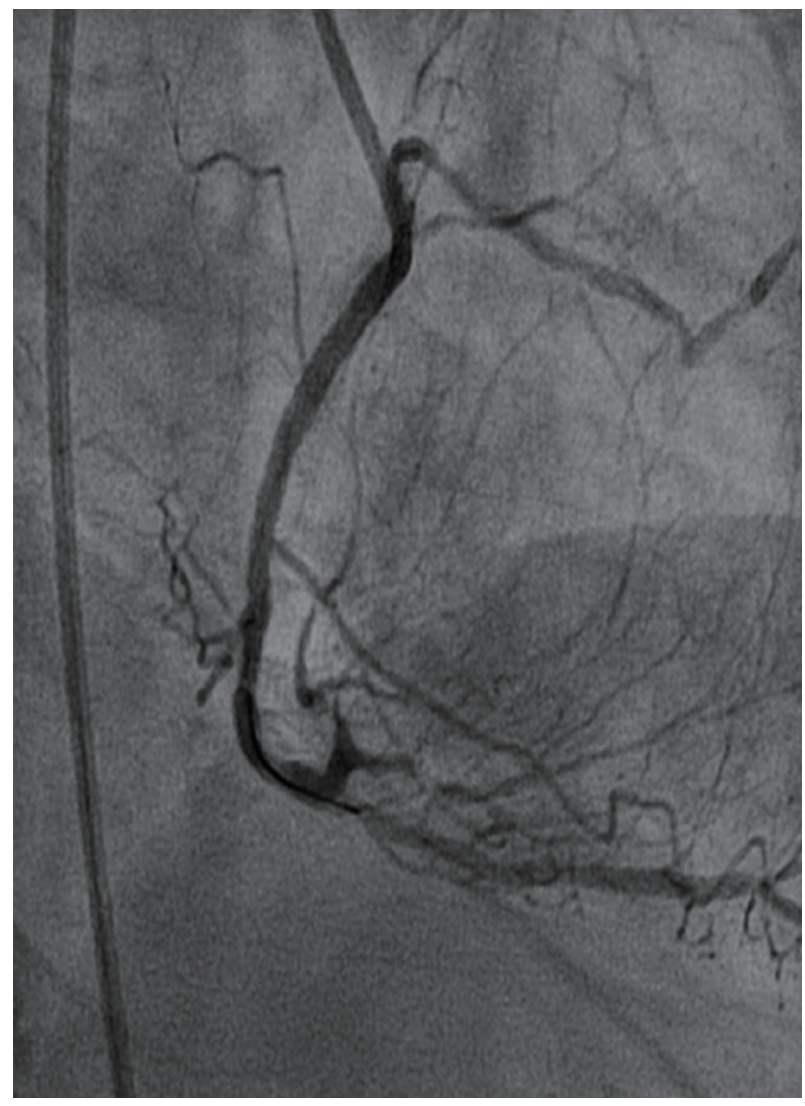

Figure 11. Coronary angiography RAO 30/CA 50 view showing good results after proximal and mid RCA stent deployment. 


\section{Discussion}

Most of the cases of SCA are asymptomatic. The malignant variety of SCA in which life-threatening symptom can occur in minor of patients is the type in which the coronary artery courses between the great vessels (aorta and pulmonary artery). Cardiac MSCT allows identifying comprehensive characterizations of coronary artery anomaly and may be useful to gain accurate insight into the culprit artery when the diagnosis and therapeutic approach are not clearly established by coronary angiography. Cardiac magnetic resonance imaging provides three-dimensional image and patient could avoid exposure to radiation. We present three rare cases of SCA in setting of ACS and culprit lesion in RCA, treated successfully with PCI.

\section{Conclusions}

Performing intervention of a SCA is a challenging situation. It requires a proper selection of instruments, well-experienced operator to perform the intervention and each case should be treated individually, according to the anatomical variations.

\section{Conflict of Interest}

The authors have no potential conflict of interest.

\section{References}

1. Walsh R, Nielsen JC, Ko HH, Sanz J, Srivastava S, Par- ness IA, Lytrivi ID. Imaging of congenital coronary artery anomalies. Pediatr Radiol. 2011;41(12):1526-1535.

2. Yamanaka O, Hobbs RE. Coronary artery anomalies in 126,595 patients undergoing coronary arteriography. Cathet Cardiovasc Diagn. 1990;21(1):28-40.

3. Arteaga RB, Tronolone J, Mandawat M. Single coronary ostium - a right coronary artery arising from the left main coronary artery. J Invasive Cardiol. 2006;18(9):E241243.

4. Frommelt PC. Congenital coronary artery abnormalities predisposing to sudden cardiac death. Pacing Clin Electrophysiol. 2009;32(Suppl 2):S63-66.

5. Angelini P, Velasco JA, Flamm S. Coronary anomalies: incidence, pathophysiology, and clinical relevance. Circulation. 2002;105(20):2449-2454.

6. Wilkins CE, Betancourt B, Mathur VS, Massumi A, De Castro CM, Garcia E, Hall RJ. Coronary artery anomalies: a review of more than 10,000 patients from the Clayton Cardiovascular Laboratories. Tex Heart Inst J. 1988;15(3):166-173.

7. Morphet JA. Congenital coronary artery anomalies: diagnosable, premortem? J Am Coll Cardiol. 2001;38(5):15871588.

8. Shriki JE, Shinbane JS, Rashid MA, Hindoyan A, Withey JG, DeFrance A, Cunningham M, et al. Identifying, characterizing, and classifying congenital anomalies of the coronary arteries. Radiographics. 2012;32(2):453-468.

9. Tariq R, Kureshi SB, Siddiqui UT, Ahmed R. Congenital anomalies of coronary arteries: Diagnosis with 64 slice multidetector CT. Eur J Radiol. 2012;81(8):1790-1797.

10. Benedek T, Gyongyosi M, Benedek I. Multislice computed tomographic coronary angiography for quantitative assessment of culprit lesions in acute coronary syndromes. Can J Cardiol. 2013;29(3):364-371. 